\title{
The impact of resource competition on neurite outgrowth
}

\author{
Johannes JJ Hjorth ${ }^{1 *}$, Jurjen Broeke ${ }^{2}$, Huib Mansvelder ${ }^{1}$, Jaap van Pelt ${ }^{1}$, Arjen van Ooyen ${ }^{1}$ \\ From Twentieth Annual Computational Neuroscience Meeting: CNS*2011 \\ Stockholm, Sweden. 23-28 July 2011
}

Axonal and dendritic growth is a highly dynamic process, where neurites elongate, bifurcate and retract. Local calcium influx [1] or local depolarisation [2] of a neurite can cause it to grow faster. The rapid growth of one branch is often accompanied by a retraction of other nearby branches [3]. This process is important for correct wiring of neuronal circuits [4]. In this study we investigate resource competition as a potential mechanism to explain the interactions between neurite branches. We have created a full compartmental model developed in python [5] using numpy [6] building on a previous modelling study [7] which suggested that the interplay between neurites might be explained by competition for resources produced in the soma; however, this has not been rigorously investigated. Neurons are stabilised by a cytoskeleton of microtubules [8]. Tubulin is produced in the soma from where it moves through diffusion and active transport to the growth cone at the tip of the neurite. At the growth cone the tubulin is polymerised, and becomes part of the microtubule cytoskeleton. In our model, neurite elongation is driven by concentration dependent tubulin polymerisation at the growth cone. We show that tubulin competition can account for the interplay between elongating and retracting neurites seen in $[1,3]$. We quantify the distance dependence of the interactions between growth cones. Furthermore we show that the model can correctly predict the growth of an experimentally recorded growth cone given the information about how the neighbouring neurites grow out. We plan to make additional experiments to test our predictions and investigate whether the estimated values for the decay,

\footnotetext{
* Correspondence: johannes.hjorth@cncr.vu.nl

${ }^{1}$ Integrative Neurophysiology, Center for Neurogenomics and Cognitive Research (CNCR), VU University Amsterdam, De Boelelaan 1085, 1081 HV Amsterdam, The Netherlands

Full list of author information is available at the end of the article
}

diffusion and active transport required for competitive effects in the model match those of tubulin.

\section{Acknowledgments}

JH was supported by NWO grant 635.100.017 awarded to Arjen van Ooyen, and the EU BIO-ICT Project SECO (216593).

\section{Author details}

${ }^{1}$ Integrative Neurophysiology, Center for Neurogenomics and Cognitive Research (CNCR), VU University Amsterdam, De Boelelaan 1085, $1081 \mathrm{HV}$ Amsterdam, The Netherlands. ${ }^{2}$ Functional Genomics, Center for Neurogenomics and Cognitive Research (CNCR), VU University Amsterdam, De Boelelaan 1085, 1081 HV Amsterdam, The Netherlands.

Published: 18 July 2011

\section{References}

1. Hutchins BI, Kalil K: Differential outgrowth of axons and their branches is regulated by localized calcium transients. J Neurosci 2008, 28:143-153.

2. Singh KK, Miller FD: Activity regulates positive and negative neurotrophin-derived signals to determine axon competition. Neuron 2005, 45:837-845.

3. Da Fontoura Costa LETM, Manoel F, Fancereau F, Chelly J, van Pelt J, Ramakers GJA: A shape analysis framework for neuromorphometry. Network: Comput in Neural Syst 2002, 13:283-310, reorganization in adult cat striate cortex. Nature 368: 737-740..

4. Dent EW, Barnes AM, Tang F, Kalil K: Netrin-1 and semaphoring 3A promote or inhibit cortical axon branching, respectively, by reorganization of the cytoskeleton. J Neurosci 2004, 24:3002-3012.

5. van Rossum G, Drake FL: Python Reference Manual. PythonLabs, Virginia, USA; 2001 [http://www.python.org].

6. Ascher D, Dubois PF, Hinsen K, Hugunin J, Oliphant T: Numerical Python. Lawrence Livermore National Laboratory, Livermore, California, USA; 2001 [http://www.pfdubois.com/numpy].

7. van Ooyen A, Graham BP, Ramakers GJA: Competition for tubulin between growing neurites during development. Neurocomputing 2001, 38-40:73-78.

8. Conde C, Caceres A: Microtubule assembly, organization and dynamics in axons and dendrites. Nature Rev Neurosci 2009, 10:319-332.

doi:10.1186/1471-2202-12-S1-P353

Cite this article as: Hjorth et al:: The impact of resource competition on neurite outgrowth. BMC Neuroscience 2011 12(Suppl 1):P353.
(C) 2011 Hjorth et al; licensee BioMed Central Ltd. This is an open access article distributed under the terms of the Creative Commons Attribution License (http://creativecommons.org/licenses/by/2.0), which permits unrestricted use, distribution, and reproduction in any medium, provided the original work is properly cited. 